\title{
Stability of the sperm plasma membrane of hibernating bats (Myotis velifer) compared with other mammals
}

\author{
E. G. Crichton ${ }^{1}$, P. H. Krutzsch ${ }^{2}$ and R. Yanagimachi ${ }^{3}$ \\ ${ }^{1}$ Department of Molecular and Cell Biology, Penn State University, University Park, PA 16802, \\ USA; ${ }^{2}$ Department of Anatomy, University of Arizona, Tucson, AZ 85724, USA; and \\ ${ }^{3}$ Department of Anatomy and Reproductive Biology, University of Hawaii, 1951 East West Road, \\ Honolulu, HI 96822, USA
}

\begin{abstract}
Previous experiments have established that the long-lived spermatozoa of hibernating bats are resistant to the acrosome reaction and fertilization in vitro using conventional techniques. We tested the hypothesis that the membranes of these spermatozoa are more resistant to perturbation than those of other mammals. We exposed them to non-specific bilayer destabilizing agents and abrupt changes in incubation temperature and tested their response by observing their status (motility and viability) after a time interval compared with other mammals (golden hamster, rabbit, human). The results did not support the hypothesis. The inherent longevity of bat spermatozoa may thus be a function of some component other than unique resilience of their plasma membrane.
\end{abstract}

\section{Introduction}

It has long been known that the spermatozoa of many species of bats are stored for many months, their prolonged viability far exceeding that of any other mammals (Wimsatt, 1942, 1944; Racey, 1973, 1975, 1979). They are stored in the caudae epididymides of males as well as in the uterus and uterotubal junction of females (Hiraiwa and Uchida, 1956a, b; Krutzsch et al., 1982; Uchida and Mori, 1987). For most of the bat species displaying this phenomenon, storage largely coincides with hibernation, but limited storage of spermatozoa has also been described in some tropical heterothermic species that do not hibernate (Gopalakrishna and Madhavan, 1971; Medway, 1972). Thus, low temperature cannot be solely responsible for the unusual longevity of bat spermatozoa. Either the spermatozoa themselves or their storage organs must be modified in some way to provide for this unique situation.

Research has sought to understand the biology of sperm longevity in bats by morphological (Fawcett and Ito, 1965; Uchida and Mori, 1972; Racey, 1975; Krutzsch et al., 1982; Mori et al., 1982; Uchida et al., 1984), biochemical (Crichton et al., 1981, 1982; Krutzsch et al., 1984) and experimental (Lambert, 1981; Uchida and Mori, 1987) approaches. However, although several theories have been offered to explain sperm storage, none has been unequivocally substantiated.

We have established that bat spermatozoa resist capacitation and the acrosome reaction when cultured under conditions (Yanagimachi, 1988) that are known to induce these events in other mammals; similar observations were made after experimental variations upon established procedures (Krutzsch and Crichton, 1991). Furthermore, contrary to the observations of Lambert (1981), in vitro fertilization tests using the hamster zona-pellucida-free oocyte test failed to reveal decondensing

Received 21 August 1991 bat sperm heads within hamster ooplasm, even after exposure to the calcium ionophore $\mathrm{A} 23187$ and various proteases. That a normal mammalian acrosome reaction does occur in bat spermatozoa, however, is shown by the occurrence of vesicular membranes, on occasion, after the exposure of spermatozoa to the calcium ionophore A23187 or to bat oocyte-cumulus complexes. Together these observations suggested that further attempts to produce in vitro fertilization with bat spermatozoa should be suspended until more knowledge accumulates on the underlying mechanisms for their unique longevity.

The unusual longevity, 'stability' and resistance of bat spermatozoa to in vitro fertilization (IVF) could be due to (i) unique resilience of the plasma membrane of bat spermatozoa, thereby eliminating preparation of the sperm surface before effective interaction with the oocyte or (ii) failure to provide a critical factor(s) in the incubation medium. A unique species specificity of the hamster oocyte may lead to exclusion of bat spermatozoa. To test the first possibility, a series of experiments was performed to test the tolerance of bat spermatozoa to environmental conditions that would perturb their plasma membrane. We compared the viability of bat spermatozoa with that of the hamster, human and rabbit in various in vitro conditions, to assess whether bat spermatozoa are unique in their response.

\section{Materials and Methods}

\section{Culture media}

BWW medium (Biggers et al, 1971) containing 0.3\% bovine serum albumin (BSA: Fraction V, Sigma, St Louis, MO) was used for all experiments. Unless otherwise stated, all chemicals were purchased from Mallinckrodt Chemical Company (St Louis, MO) or Sigma Chemical Company (St Louis, MO). 
Table 1. Minimum concentration of various membrane-destabilizing reagents at which $100 \%$ of spermatozoa of four mammals were immobilized in $5 \mathrm{~min}$

\begin{tabular}{lccccc}
\hline & & \multicolumn{5}{c}{ Spermatozoa of: } \\
\cline { 3 - 6 } Reagent & $\begin{array}{c}\text { Concentration } \\
\text { range tested }\end{array}$ & Hamster & Bat & Rabbit & Human \\
\cline { 3 - 6 } & & & & & \\
Triton X-100 & $0.001-0.1 \%$ & 0.02 & 0.02 & 0.03 & 0.01 \\
Hyamine & $0.001-0.1 \%$ & 1.0 & $1-2$ & 1.0 & 1.0 \\
Lauryl sulfate & $0.1-2.0 \mathrm{mmol} \mathrm{I}^{-1}$ & 1.0 & 1.0 & 3.0 & 2.0 \\
Polymixine & $0.1-3.0 \%$ & 2.0 & 3.0 & $>3.0$ & 2.0 \\
Tetracaine & $1.0-3.0 \mathrm{mmol} 1^{-1}$ & 0.1 & 0.1 & 0.1 & 0.1 \\
Digitonin & $0.01-2.0 \%$ & 0.001 & 0.001 & 0.1 & 0.1 \\
Saponin & $0.0001-1.0 \%$ & 0.01 & 0.01 & 0.01 & 0.01 \\
Nystatin & $0.0001-1.0 \%$ & 35 & 40 & 35 & 50 \\
Acetone/methanol & $10-50 \%$ & & & & \\
\hline
\end{tabular}

\section{Collection of spermatozoa}

Adult male bats (Myotis velifer) were collected from the wild in Arizona in the autumn (September-October 1989). The testes were atrophying at this time and all the spermatozoa had been transported to the caudae epididymides where they are stored until the following spring. For several weeks after capture, animals were kept at room temperature and food (larval Tenebrio molitor) and water were provided ad libitum until they had accumulated sufficient body fat reserves to hibernate. Bats were then placed in an artificial hibernaculum $\left(15^{\circ} \mathrm{C}\right)$, where they were held until required for experiments.

Caudae epididymides were removed from etherized bats, wrapped in saline-moistened gauze within a tightly capped disposable microcentrifuge tube and placed in a styrofoam box with ice for overnight shipment to Hawaii. The inside temperature of the box was $6-10^{\circ} \mathrm{C}$ upon arrival. The tubes containing the epididymides were then placed in a refrigerator $\left(4^{\circ} \mathrm{C}\right)$ until the start of an experiment that day (i.e. about $24 \mathrm{~h}$ after collection of epididymides).

Spermatozoa were liberated from the caudae epididymides of bats, Syrian (golden) hamsters and rabbits by puncturing the epididymal tubules with a fine needle. Human ejaculated spermatozoa, which were obtained through a human IVF clinic, were washed and centrifuged $(500 \mathrm{~g}, 5 \mathrm{~min})$ to remove seminal fluids and debris. The 'swim-up' method (Overstreet et al., 1979; Cummins and Yanagimachi, 1986) was used for the collection of highly motile populations of hamster and human spermatozoa. Populations of spermatozoa (about $1-5 \times 10^{7}$ ) from all species were estimated to be at least $80 \%$ motile at the start of all experiments.

\section{Effect of temperature shock}

To examine the effect of temperature shock on sperm viability, sperm suspensions were placed in narrow glass microcentrifuge tubes (approximately $2 \mathrm{~mm}$ internal diameter, $200 \mu \mathrm{l}$ volume), and transferred rapidly between warm $\left(37^{\circ} \mathrm{C}\right)$ and cold $\left(0^{\circ} \mathrm{C}\right)$ water baths. The duration of each exposure to warm and cold temperature was 5-10 min; 1-5 successive exposures ( $2-10$ transfers) were made before spermatozoa were examined.
Effect of plasma-membrane-destabilizing agents on the survival of spermatozoa

The following membrane-perturbing reagents were prepared in BWW immediately before use:

(1) Triton X-100 (0.001-0.1\%), hyamine 2389 (Rhom and Haas Co.) $(0.001-0.1 \%)$ and lauryl sulfate $\left(0.1-2 \mathrm{mmol} \mathrm{l}^{-1}\right)$. These detergents are known to disorganize membrane phospholipids.

(2) Polymixine $(0.1-3.0 \%)$, a chemical that enters lipid bilayers and increases membrane permeability (Pache et al., 1972).

(3) Tetracaine $\left(1.0-3.0 \mathrm{mmol} \mathrm{l}^{-1}\right)$, a chemical that enters lipid bilayers to stabilize membrane lipids at low concentrations $\left(<1 \mathrm{mmol} \mathrm{l}^{-1}\right)$ and induce membrane lysis at high concentrations $\left(>1 \mathrm{mmol}^{-1}\right.$ ) (Butler et al., 1973).

(4) Digitonin $(0.01-2.0 \%)$, saponin $(0.0001-1.0 \%)$ and nystatin $(0.001-1.0 \%$ - dissolved initially in dimethyl sulfoxide), reagents that bind to sterols and disrupt membranes (Sutton et al., 1961; Kinsky et al., 1966; Addinck et al., 1972; Elliot and Haas, 1976).

(5) A 1:1 mixture of acetone and methanol (10-50\%), a lipid solvent.

Equal volumes $(15 \mu \mathrm{l})$ of a sperm suspension and a test solution were mixed and incubated together for $5 \mathrm{~min}$ at room temperature $\left(25^{\circ} \mathrm{C}\right)$. At the end of this period, sperm motility and viability were assessed.

\section{Survival (motility) of spermatozoa}

The sensitivity of sperm plasma membranes to the chemicals tested was evaluated by determining the minimum concentration at which $100 \%$ of spermatozoa were immobilized in $5 \mathrm{~min}$. The survival of spermatozoa was assessed subjectively by viewing the drops of sperm suspension using phase-contrast or dark-field microscopy. Viability of immobilized spermatozoa was tested by mixing equal volumes of a $1 \%$ solution of Trypan Blue in Dulbecco's phosphate-buffered saline and the sperm suspension on a glass slide and immediately counting the proportion of live (unstained) to dead (stained) cells. At least 
three replicates of each experiment were run using different males.

\section{Results}

\section{Resistance of spermatozoa to temperature shifts}

Rapid transfer of sperm samples between cold $\left(0^{\circ} \mathrm{C}\right)$ and warm $\left(37^{\circ} \mathrm{C}\right)$ temperatures did not greatly affect the viability of bat, rabbit or human spermatozoa. At least $70 \%$ of these spermatozoa were motile and alive even after exposure for five times to each set of temperatures (10 transfers). Under the same conditions, no more than $25 \%$ of hamster spermatozoa were alive.

\section{Resistance of spermatozoa to membrane-destabilizing agents}

Bat spermatozoa showed no greater tolerance to membraneactive reagents than the spermatozoa of the other mammals tested (Table 1). They were immobilized at similar or even lower concentrations.

\section{Discussion}

In the present study, we looked to the plasma membrane as a possible source of difference between spermatozoa of bats and those of other mammals. It is known that the lipid composition of the sperm plasma membrane differs considerably among species (Darin-Bennett and White, 1977; Peterson and Russell, 1985; Hinkovska et al., 1986; Nikolopoulou et al., 1986; Parks et al., 1987; Agrawal et al., 1988). It appears that the cholesterol:phospholipid ratio of the sperm plasma membrane may underlie species-specific differences in resistance to environmental stresses such as cold shock (Wales and White, 1959; Darin-Bennett and White, 1965; Darin-Bennett et al., 1974). The time needed for capacitation of bat spermatozoa may be influenced by the lipid composition of the sperm plasma membrane, since the removal of cholesterol from the sperm plasma membrane has been implicated as an important step in this process in other species (Davis, 1978, 1981; Davis et al., 1979; Go and Wolf, 1983; Langlais and Roberts, 1985; Suzuki and Yanagimachi, 1989; Parks and Ehrenwald, 1990).

The results of this study did not support our hypothesis that bat spermatozoa, by virtue of their inherent longevity, their ability to resist extreme temperature conditions in vivo and their resistance to our attempts to induce the acrosome reaction in vitro, are more tolerant to environmental stresses than are the spermatozoa of other mammals. Although bat spermatozoa displayed a much greater resilience than hamster spermatozoa to the imposition of all (except saponin) membrane-destabilizing agents and abrupt temperature shifts, they performed no better than rabbit or human spermatozoa under all experimental conditions tested. No explanation can be offered for the greater susceptibility of hamster and bat spermatozoa to saponin; there is no information for the membrane-destructive effects of this detergent that might distinguish between plasma membrane components. The observation that both human and rabbit spermatozoa showed a high degree of tolerance to temperature shock is consistent with the data of Wales and White (1959). Human and rabbit spermatozoa have a higher cholesterol content (Darin-Bennett and White, 1977) than do spermatozoa of mammals that are more susceptible to temperature shock (e.g. bull, ram).

Despite our failure to demonstrate a difference between the spermatozoa of bats and those of other mammals under the conditions tested, it remains apparent, from our IVF trials, that bat spermatozoa are unique. Although it is possible that zona-free hamster oocytes are not penetrated by bat spermatozoa, it is apparent from electron microscopy that the acrosome reaction of bat spermatozoa is difficult to induce in vitro, at least under conditions that are sufficient for most other species. Capacitation, the acrosome reaction and egg penetration may require the provision of some critical component in the medium that has yet to be identified for this system. Mate and Rodger (1991) have noted a similar stability of the sperm acrosome of some marsupial species which also resist efforts to induce the acrosome reaction.

Although the present study failed to demonstrate a uniqueness of bat spermatozoa, the conditions and agents used may have failed to detect delicate, yet important, differences in membrane characteristics between bat spermatozoa and those of other mammals. Accordingly, studies to measure fine changes in sperm biology before immobilization are in progress, using rheological and flow cytometry technology.

Within the limitations of the data collected, the results of these experiments suggest that the spermatozoa of hibernating, sperm-storing bat species may not be modified in terms of the lipid composition of their plasma membrane. Rather, their prolonged viability may reflect the presence of some other membrane specialization (e.g. a glycoprotein) that must be removed or altered during modification of the plasma membrane for fertilization (Yanagimachi, 1988). Alternatively, specialized conditions in the storage sites in the male and female reproductive tracts may act in some way to maintain the life of the spermatozoa of sperm-storing species of bats.

This work was supported by NSF-RR-8903125 to E. G. Crichton.

\section{References}

Addink ADF, Boer P, Wakabayashi T and Green DE (1972) Enzyme localization in beef-heart mitochondria Eurpoean Journal of Biochemistry 29 47-59

Agrawal P, Magargee SF and Hammerstedt RH (1988) Isolation and characterization of the plasma membrane of rat cauda epididymal spermatozoa Journal of Andrology 9 178-189

Biggers JD, Whitten WK and Whittingham DG (1971) The culture of mouse embryos in vitro. In Methods of Mammalian Embryology pp 93-116 Ed. IC Daniel. Freeman, San Francisco

Butler KW, Schneider $\mathrm{H}$ and Smith ICP (1973) The effects of local anesthetics on lipid multilayers. A spin probe study Archives of Biochemistry and Biophysics 154 548-554

Crichton EG, Krutzsch PH and Wimsatt WA (1981) Studies on prolonged spermatozoa survival in Chiroptera. I. The role of uterine free fructose in the spermatozoa storage phenomenon Comparative Biochemistry and Physiology 70A $387-395$

Crichton EG, Krutzsch PH and Chvapil M (1982) Studies on prolonged spermatozoa survival in Chiroptera. II. The role of zinc in the spermatozoa storage phenomenon Comparative Biochemistry and Physiology 71A 71-77

Cummins JM and Yanagimachi R (1986) Development of ability to penetrate the cumulus oophorus by hamster spermatozoa capacitated in vitro in relation to the timing of the acrosome reaction Gamete Research 15 187-212 
Darin-Bennett A and White IG (1965) Cholesterol and phospholipid content of mammalian spermatozoa and its relation to membrane structure and cold-shock Experimental Cell Research 38 217-246

Darin-Bennett A and White IG (1977) Influence of the cholesterol content of mammalian spermatozoa on susceptibility to cold shock Cryobiology 14 466-470

Darin-Bennett A, Poulos A and White IG (1974) The phospholipids and phospholipid-bound fatty acids and aldehydes of dog and fowl spermatozoa Joumal of Reproduction and Fertility 41 471-474

Davis BK (1978) Inhibition of fertilizing capacity in mammalian spermatozoa by natural and synthetic vesicles. In Symposium on Pharmacological Effects of Lipids. American Oil Chemists' Society Monograph No. 5 pp 145-157 Ed. JJ Kabara. American Oil Chemists' Society, Champaign, IL

Davis BK (1981) Timing of fertilization in mammals: sperm cholesterol/ phospholipid ratio as a determinant of the capacitation interval Proceedings of the National Academy of Sciences, USA 78 7560-7564

Davis BK, Byrne R and Hungund B (1979) Studies on the mechanism of capacitation. II. Evidence for lipid transfer between plasma membrane of rat sperm and serum albumin during capacitation in vitro Biochimica et Biophysica Acta $558257-266$

Elliot WB and Haas DW (1967) Preparation and properties of digitonin particles from beef heart Methods in Enzymology 10 179-181

Fawcett DW and Ito S (1965) The fine structure of bat spermatozoa American Joumal of Anatomy 116 567-610

Go KJ and Wolf DP (1983) The role of sterols in sperm capacitation Advances in Lipid Research 20 317-330

Gopalakrishna A and Madhavan A (1971) Survival of spermatozoa in the female genital tract of the Indian vespertilionid bat, Pipistrellus ceylonicus chrysothrix (Wroughton) Proceedings of the Indian Academy of Sciences 73 43-49

Hinkovska VT, Dimitrov GP and Koumanov KS (1986) Phospholipid composition and phospholipid asymmetry of ram spermatozoa plasma membranes Intermational Journal of Biochemistry 18 1115-1121

Hiraiwa YK and Uchida TA (1956a) Fertilization in the bat, Pipistrellus abramus abramus (Temminck). III. Fertilizing capacity of spermatozoa stored in the uterus after copulation in the fall Scientific Bulletin of the Faculty of Agriculture, Kyushu University 15 565-574

Hiraiwa YK and Uchida TA (1956b) Fertilization in the bat, Pipistrellus abramus. A successful example of artificial insemination with epididymal spermatozoa in autumn Science (Kagata), Tokyo 26535

Kinsky SC, Luse SA and van Deenen LLM (1966) Interaction of polyene antibiotics with natural and artificial membrane systems Federal Proceedings 25 1503-1510

Krutzsch PH and Crichton EG (1991) Fertilization in Bats. In A Comparative Overview of Mammalian Fertilization pp 137-149 Eds BS Dunbar and M O'Rand. Plenum Press, New York

Krutzsch PH, Crichton EG and Nagle RB (1982) Studies on prolonged spermatozoa survival in Chiroptera. A morphological examination of storage and clearance of intrauterine and caudal epididymal spermatozoa in the bat Myotis lucifugus and $M$. velifer American Joumal of Anatomy 165 421-434

Krutzsch PH, Crichton EG, Lennon DLF, Stratman FW and Carter A (1984) Studies on prolonged spermatozoa survival in Chiroptera. III. Preliminary data on carnitine Andrologia 16 34-37

Lambert H (1981) Temperature dependence on capacitation in bat sperm monitored by zona-free bat ova Gamete Research 4 525-533

Langlais J and Roberts KD (1985) A molecular membrane model of sperm capacitation and the acrosome reaction of mammalian spermatozoa Gamete Research 12 183-224

Mate KE and Rodger JC (1991) Stability of the acrosome of the brush-tailed possum (Trichosurus vulpecula) and tammar wallaby (Macropus engenii) in vitro and after exposure to conditions and agents known to cause capacitation or acrosome reaction of eutherian spermatozoa Journal of Reproduction and Fertility 914 I-48

Medway L (1972) Reproductive cycles of the flat-headed bats Tylonycteris pachypus and T. robustula (Chiroptera, Vespertilionidae) in a humid equatorial environment Zoological Journal Linnaean Society 51 33-61

Mori T, Oh YK and Uchida TA (1982) Sperm storage in the oviduct of the Japanese greater horseshoe bat, Rhinolophus ferrum-equinum nippon Journal of the Faculty of Agriculture, Kyushu University 27 47-55

Nikolopoulou M, Soucek DA and Vary JC (1986) Modulation of the lipid composition of boar sperm plasma membranes during an acrosome reaction in vitro Archives of Biochemistry and Biophysics 250 30-37

Overstreet JW, Yanagimachi R, Katz DF, Ilayashi K and Hanson FW (1979) Penetration of human spermatozoa into the human zona pellucida and the zona-free hamster eggs: a study of fertile donors and infertile patients fertility and Sterility 33 534-542

Pache W, Chapman D and Hillaby R (1972) Interaction of antibiotics with membranes. Polymixine B and gramicidin S Biochimica et Biophysica Acta 250 358-364

Parks JE and Ehrenwald E (1990) Cholesterol efflux from mammalian sperm and its potential role in capacitation. In Fertilization in Mammals pp 155-167 Eds BD Bavister, J Cummins and ERS Roldan. Serono Symposia, SA, Norwell, MA

Parks JE, Arion JW and Foote RH (1987) Lipids of plasma membrane and outer acrosomal membrane from bovine spermatozoa Biology of Reproduction 37 $1249-1258$

Peterson RN and Russell D (1985) The mammalian spermatozoon: a model for the study of regional specificity in plasma membrane organization and function Tissue and Cell 17 769-791

Racey PA (1973) The viability of spermatozoa after prolonged storage by male and female European bats Periodicum biologorum 775 201-205

Racey PA (1975) The prolonged survival of spermatozoa in bats. In The Biology of the Male Gamete pp 385-416 Eds JG Duckett and PA Racey. Academic Press, London

Racey PA (1979) The prolonged storage and survival of spermatozoa in Chiroptera Journal of Reproduction and Fertility 56 391-402

Sutton DD, Arnow PM and Lampen JD (1961) Effect of high concentration of nystatin upon glycolysis and cellular permeability in yeast Proceedings of the Society for Experimental Biology and Medicine 108 170-175

Suzuki F and Yanagimachi $\mathbf{R}(1989)$ Changes in the distribution of intramembranous particles and filipin-reactive membrane sterols during in vitro capacitation of golden hamster spermatozoa Gamete Research 23 335-347

Uchida TA and Mori T (1972) Electron microscopic analysis of the mechanisms of fertilization in Chiroptera. I. Acrosomal reaction and consequence to death of the sperm in the Japanese long-fingered bat, Miniopterus schreibersii fuliginosus Scientific Bulletin of the Faculty of Agriculture, Kyushu University 28 $177-184$

Uchida TA and Mori T (1987) Prolonged storage of spermatozoa in tibernating bats. In Recent Advances in the Study of Bats pp 351-356 Eds MB Fenton, PA Racey and JMV Rayner. Cambridge University Press, Cambridge

Uchida TA, Mori T and Oh YK (1984) Sperm invasion of the oviducal mucosa, fibroblastic phagocytosis and endometrium sloughing in the Japanese greater horseshoe bat, Rhinolophus ferrum equinum nippon Cell and Tissue Research $236327-331$

Wales RG and White IG (1959) The susceptibility of spermatozoa to temperature shock Journal of Endocrinology 19 211-220

Wimsatt WA (1942) Survival of spermatozoa in the female reproductive tract of the bat Anatomical Record 83 299-307

Wimsatt WA (1944) Further studies on the survival of spermatozoa in the female reproductive tract of the bat Anatomical Record 88 193-204

Yanagimachi R (1988) Mammalian fertilization. In Physiology of Reproduction pp 135-185 Eds E Knobil and I Neil. Raven Press, New York 